\title{
Wind turbine wake models' evaluation for different downstream locations
}

\author{
Panagiotis Triantafyllou (D) and John K. Kaldellis \\ Laboratory of Soft Energy Applications \& Environmental Protection, Department of Mechanical Engineering, \\ University of West Attica, 12241, Egaleo-Athens, Greece
}

Received: 6 July 2021 / Received in final form: 14 October 2021 / Accepted: 15 October 2021

\begin{abstract}
The land use limitations, especially for onshore applications, have led modern Wind Turbines (WTs) to be aggregated in wind parks under the scope of minimizing the necessary area required. Within this framework, the trustworthy prediction of the wind speed deficiency downstream the WTs' hub (known also as the "wake effect") and the meticulous wind park micrositing are of uppermost importance for the optimized WTs siting across the available land area. In this context, substantial effort has been made by the academic and research community, contributing to the deployment of several analytical, numerical and semi-empirical wake models, attempting to estimate the wind speed values at different locations downstream a WT. The accuracy of several semi-empirical and analytical wake models, serving also as the basis for pertinent commercial software development, is investigated in the present work, by comparing their outcome with experimental data from a past research work that concerns the wake flow. The dimensionless streamwise distance (known also with the term "downstream distance") from the WT's hub is used as benchmark in order to categorize and evaluate the calculation results. A dedicated comparison between the wind speed cases investigated is conducted, striving to properly assess the wake models' prediction accuracy. The notable findings obtained for the wake models examined designate the requirement for subsequent research to enlighten the wake effect dynamic behavior.
\end{abstract}

\section{Introduction}

The constant environmental degradation along with the imminent carbon containing fuel reserves depletion underline the need to deploy more environmentally friendly energy resources [1] to deal with the constantly growing energy demand of the planet [2]. Under the specific scope, industrial scale wind farm clusters have been developed [3]. To this end, Wind Farm Layout Optimization (WFLO) or wind farm micrositing is crucial as it defines the wind farm's performance, both in terms of energy yield and cost. This can be considered as a quest for the optimized Wind Turbines' (WTs) allocation across the available territory and is mainly carried out as a compromise between the optimal exploitation of the available wind energy potential of the area considered for the project implementation and the mechanical loading on downstream WTs $[4,5]$. The area ground morphology, the WT installation positions, the local electricity grid infrastructure, the installation site accessibility, the project acceptability by the local society, the wind energy

\footnotetext{
* e-mail: ptriantafyllou@uniwa.gr
}

potential quality (in terms of the wind speed values and the predominant wind directions) along with the wake effect play a principal role for this scope [6,7].

With regards to the wake effect, both energy extraction as well as radial and axial pressure drop occur as wind crosses a WT's rotor, increasing the wind speed and the wind energy deficit as a result of the relevant turbulence and airflow vortex increase downstream the WT's rotor hub (Fig. 1) [5,8].

Moreover, the mechanical fatigue on downstream WTs is intensified by the wake effect as a result of its mix with the faster-moving air that surrounds the downstream area, thus decreasing gradually their technical availability [10-13]. The sound refraction processes and the WT-generated noise propagation are also influenced by the wake effect along with the nature of the Atmospheric Boundary Layer (ABL) [14-17]. High turbulence inflow can also impact downstream WTs, even under low turbulence conditions for the upstream ones [18].

For contemporary wind energy applications, a differentiation exists between the design considerations for onshore and offshore applications. More precisely, for onshore applications, the common practice takes into account a span of around three rotor diameters for WTs installed perpendicular to the predominant wind direction 


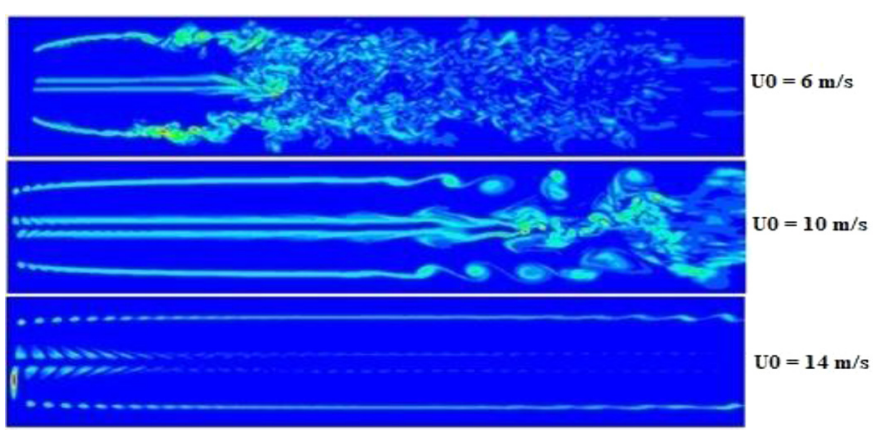

Fig. 1. Wake evolution downstream a WT's hub (adapted from [9]).

and four to six rotor diameters as an inter-row spacing [19]. For offshore projects, the current industry principle for the distance between WTs installed perpendicular to the prevalent wind direction is four to nine rotor diameters, while a distance of six to eleven rotor diameters is considered between WTs' rows [20].

Although the wake effect impact is gradually decreased with the downstream distance, a remarkable wind energy deficit usually exists up to twenty diameters $(20 \times \mathrm{D})$ distance from a preceding WT row. Hence, it is extremely important to investigate its inherent characteristics. In this context, several numerical and analytical wake models have been developed as supporting accessories by the scientific community, as a result of a perpetual effort for the optimum pre-appraisal of a potential installation site. The wake effect characteristics are typically distinguished between two discrete regions using the criteria of the downstream distance from the WT rotor (" $\times / D$ "), that is the near-wake (extending up to three " $\times / D$ ") and the far-wake (from four to twenty " $\times / \mathrm{D}$ ") region. The last region can be divided further into the mid-wake (four to nine " $\times / \mathrm{D}$ ") and the distant-wake (ten to twenty " $\times /$ D") sub-region (Fig. 2) [21].

The majority of analytical wake models do not present reliable performance with regards to the wake evolution in the near-wake region, as intense airflow swirl is dominant in the specific area $[22,23]$. In the same context, WTs are on rare occasions being positioned in downstream distances greater than ten " $\times / D$ " due to local topography, ground morphology and land availability issues. It is mainly the mid-wake sub-region that captivates the interest of both project developers and academic researchers. Nevertheless, there exist established references in the literature for a diversified wake models' outcome in the two different far-wake sub-regions. Hence, under the scope of the present work, the entire far-wake region is finally investigated.

The WTs' downstream distance and the upstream wind speed variation are considered of paramount importance for the wake effect development [24-29]. In this framework, the current work conducts a comparative assessment of eight prominent semi-empirical and analytical wake models, many of which are utilized till date as the basis for pertinent commercial software deployment. To be more specific, Section 2 attempts an introduction on the wake models classification, whereas Section 3 presents the evaluation methodology adopted. Sections 4 and 5

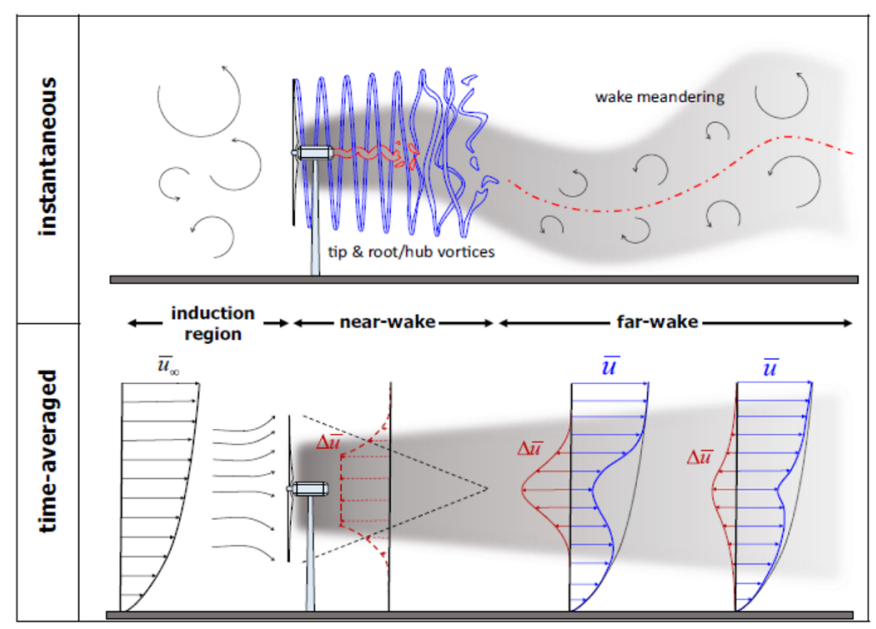

Fig. 2. Schematic representation of the two discrete wake regions downstream a WT's hub [21].

accomplish a comprehensive evaluation of the wake models' outcome on the basis of experimental data from a past research work, with both the upstream wind speed and the downstream distance diversification serving as the comparison criteria [30]. The useful conclusions drawn set the groundwork for future research activity that could be conducted in the specific scientific domain.

\section{Wake effect models classification}

The existing wake models present great variations in terms of the precision of the wind speed prediction and the computational time required for their calculation process. Presently, the wake models are principally classified in three major categories based on their intrinsic methodology:

- Linearized or non-linearized Reynolds-Averaged Navier Stokes (RANS) implemented in Computational Fluid Dynamics (CFD) software, where basic fluid equations are being integrated through diversified numerical techniques.

- Large Eddy Simulations (LES), discerned for the direct calculation of large and parameterized eddies [31].

- Empirical or semi-empirical, numerical (or analytical) models, being principally comprised of deterministic equations that characterize the conservation of flow properties and hence having the additional value of providing fundamental understanding of the physics [21].

Among the aforementioned three categories, more elaborate results can indisputably be generated by CFD and LES models in comparison to the numerical or the semi-empirical ones. However, their execution could be characterized, at least for precursory micrositing studies, by impermissible calculation time burden, considering the volume of the relevant input data required to be processed (the numerical wake models have three-to-four orders of magnitude less Central Processing Unit (CPU) time than the CFD and LES models) [21,32]. This fact renders their implementation quite difficult. In their turn, numerical wake models consider only the average or the outlier of the turbulence intensity profile and not its entire profile 


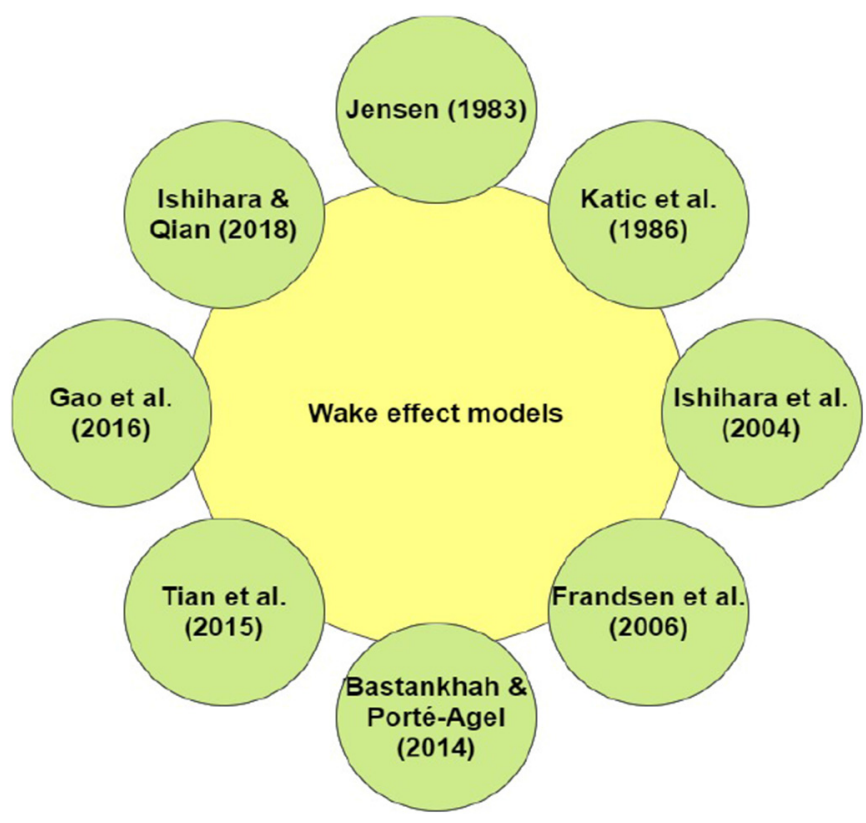

Fig. 3. Schematic representation of the wake models investigated.

variation in the radial wake direction [17]. Nevertheless, their dominant characteristics of low computational burden and simplicity consist the main drivers for their vast application as the basis for the deployment of wind parks' micrositing commercial software $[8,33,34]$. To be more precise, it is the Katic et al. and the Jensen wake models that are mostly utilized for the commercial software development, with the WAsP, WindPRO, WindSim, WindFarmer and OpenWind software being characteristic examples $[8,35]$.

Finally, an explicit distinction has to be made between the wake effect being originated from WTs of the same wind farm and these installed in adjacent wind farms. In this context, to examine the wakes orginated from an aggregate of upstream WTs (multi-wakes), more sophisticated analysis is required [21], resulting from the fact that interacting wakes can have a more notable impact on the wind speed in comparison to the sum of independent wakes [36]. The abovementioned wake overlapping or superposition effect has consisted the study object of various researchers with profound experience in the field such as Tian et al. [8], Porté-Agel et al. [21], Sun and Yang [33], Parada et al. [34], Machefaux et al. [37] and Gaumond et al. [38].

\section{Evaluation methodology}

\subsection{Wake models input parameters}

The eight numerical wake models that have been investigated in the current research work along with the corresponding year of their development are presented in Figure 3.
Each of the wake models investigated uses different inputs. Among them, the Jensen wake model, owing to its simplicity (only four parameters are used as inputs), is considered the most commonly adopted model. Other more complex wake models, such as the ones of Bastankhah \& Porté-Agel and Ishihara \& Qian, also exist and take into account more specialized parameters. More information on the input parameters used by each wake model investigated can be found in Kaldellis et al. [24].

\subsection{Comparison basis}

To assess the prediction efficiency of the wake models investigated, a dedicated data set has been used as the comparison basis, being characterized by specific WT characteristics and site conditions. More precisely, experimental measurements for wind speed values in the range between 4 and $18 \mathrm{~m} / \mathrm{s}$ and for downstream distances up to twenty " $\mathrm{x} / \mathrm{D}$ " were gathered from a LiDAR device installed donwstream an Areva M5000-116 hub (cut-in wind speed value equal to $5 \mathrm{~m} / \mathrm{s}$ and rated wind speed value equal to $12 \mathrm{~m} / \mathrm{s}$ ) [30]. The experimental data compiled were divided in bins on the basis of a $2 \mathrm{~m} / \mathrm{s}$ step, beginning from the $[4-6] \mathrm{m} / \mathrm{s}$ bin till the [16-18] $\mathrm{m} / \mathrm{s}$ bin. To facilitate the subsequent assessment, the middle point of each considered wind speed bin is used.

\subsection{Percentage deviation calculation}

The wake models' performance percentage deviation (found also in the literature with the term "error rate" [39]) from the relevant data is used as the basis for the assessment procedure conducted, with the downstream distance being used as the comparison parameter. The specific deviation is calculated based on equation (1):

$$
\text { Deviation }(\%)=\frac{\left|V_{\text {deficit,model }}-V_{\text {deficit,reference }}\right|}{V_{\text {deficit,reference }}} \times 100 \%
$$

where $V_{\text {deficit,model }}$ is the wind speed deficit computed by each numerical wake model investigated. $V_{\text {deficit,reference }}$ is the relevant wind speed deficit retrieved from the experimental measurements.

Moreover, one can use equation (2) to calculate the pertinent average percentage deviation:

$$
\text { Average deviation }(\%)=\sum_{\frac{x}{D}=n_{1}}^{\frac{x}{D}=n_{2}} \text { Error deviation }(\%)
$$

where: $n_{1}$ is the lower bound of the examined wake subregion each time and $n_{2}$ is the upper bound of the examined wake sub-region each time.

It is worthwhile to mention that the percentage deviation in the aforementioned equations is considered in the wake center, that is when the wake radius is equal to zero, under the scope of investigating the greatest wind speed deficit condition. Hence, the investigation carried out can be translated as a worst-case scenario analysis. 


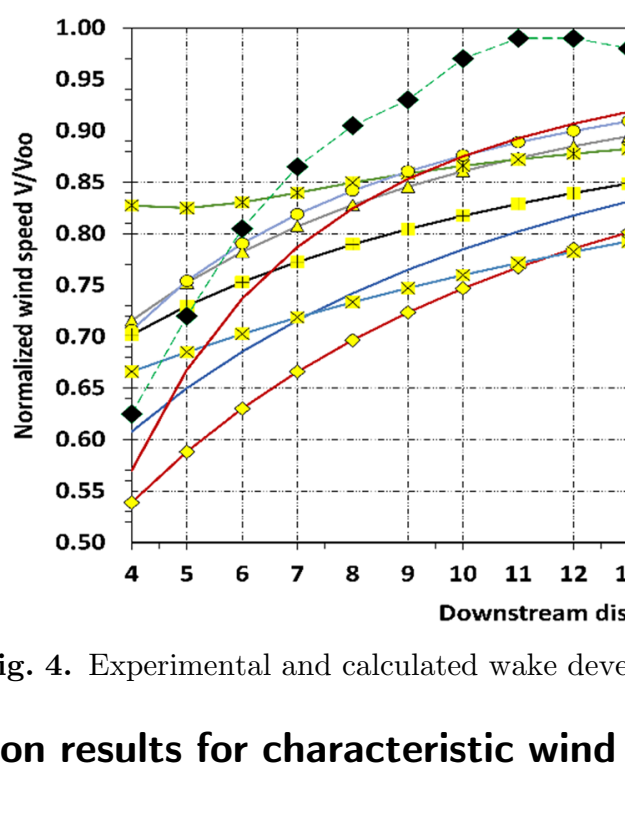

\section{Calculation results for characteristic wind speeds}

To assess the prediction efficiency of the numerical wake models examined, the relevant deviation between the experimental measurements and the calculation results for three pre-specified upstream wind speed values was initially selected to be investigated. More precisely, the cut-in wind speed value (equal to $5 \mathrm{~m} / \mathrm{s}$ ), the most approximate to the rated wind speed value (equal to $13 \mathrm{~m} / \mathrm{s}$ ) and the maximum wind speed value for which experimental measurements have been gathered (equal to $17 \mathrm{~m} / \mathrm{s}$ ), that belongs to the WT's power curve constant power segment, are examined. Figures 4-12 describe the wake models' output and their pertinent average percentage deviations for the abovementioned upstream wind speed values.

\subsection{Prediction performance evaluation for wind speed value equal to $5 \mathrm{~m} / \mathrm{s}$}

Most of the wake models investigated are characterized by acceptable percentage deviations (Fig. 4) from the experimental measurements (comparison basis data) for downstream distance in the range between five and seven " $\mathrm{x} / \mathrm{D}$ " (belonging in the mid-wake region and normally constituting the most likely location for the positioning of the second WTs' row) and in the range between fourteen and seventeen " $\mathrm{x} / \mathrm{D}$ ". Actually, for characteristic downstream distances, percentage deviations even less than $3 \%$ are recorded. Another important outcome for most of the wake models investigated is that their percentage deviation increases with the downstream distance increase in the mid-wake sub-region, whereas a reverse behaviour can be noted in the distant-wake sub-region.

In the distinct limit between the two sub-regions (downstream distance value equal to ten "x/D"), an average percentage deviation approximating $15 \%$ is noted, with the respective percentage deviations being in the range [10-23\%]. The importance of this remark can be highlighted by the key role that the WT's cut-in wind speed plays in its controller's operation, as it represents the undermost bound considered for the relevant energy generation calculations that accompany each wind power project's economic evaluation. It is also characteristic that for most of the wake models investigated, except the ones of Katic et al. and Ishihara et al., small discrepancies (in the order of less than $2 \%$ ) exist between the average percentage deviation noted in the mid-wake and the distant-wake sub-regions (Fig. 5). The greatest difference, exceeding $6 \%$, is recorded for the Katic et al. model.

The WT-generated turbulence is prevalent in the farwake region as a result of the low upstream wind speed value, hence rendering the wind flow unstable. Among the wake models investigated, only three present an adequate average percentage deviation. More precisely, for the entire far-wake region, the average percentage deviation of the Frandsen et al., the Ishihara \& Qian and the Bastankhah \& Porté-Agel models (Fig. 6) is calculated less than or equal to $8 \%$. Contrarily, the greatest average percentage deviation is attributed to the Katic et al. model, with a value outreaching $16 \%$.

\subsection{Prediction performance evaluation for wind speed value equal to $13 \mathrm{~m} / \mathrm{s}$}

The outcome of all wake models investigated was less than the rated wind speed value. Hence, acknowledging that the wind speed value case examined is near to the rated wind speed value renders the present assessment critical for the WT controller's operation and the power regulation scheme to be adopted. Except for downstream distance in the range between four and six " $\mathrm{x} / \mathrm{D}$ ", most of the wake models investigated are characterized by acceptable percentage deviations from the experimental measurements (Fig. 7), being less than $8 \%$. Furthermore, the wake models' output divergence from the experimental measurements decreases with the downstream distance increase, underlining the quite qood representation of the experimental measurements. In the same context, only the Katic et al. and the Tian et al. models are characterized by excessive percentage deviation in the distinct limit between 


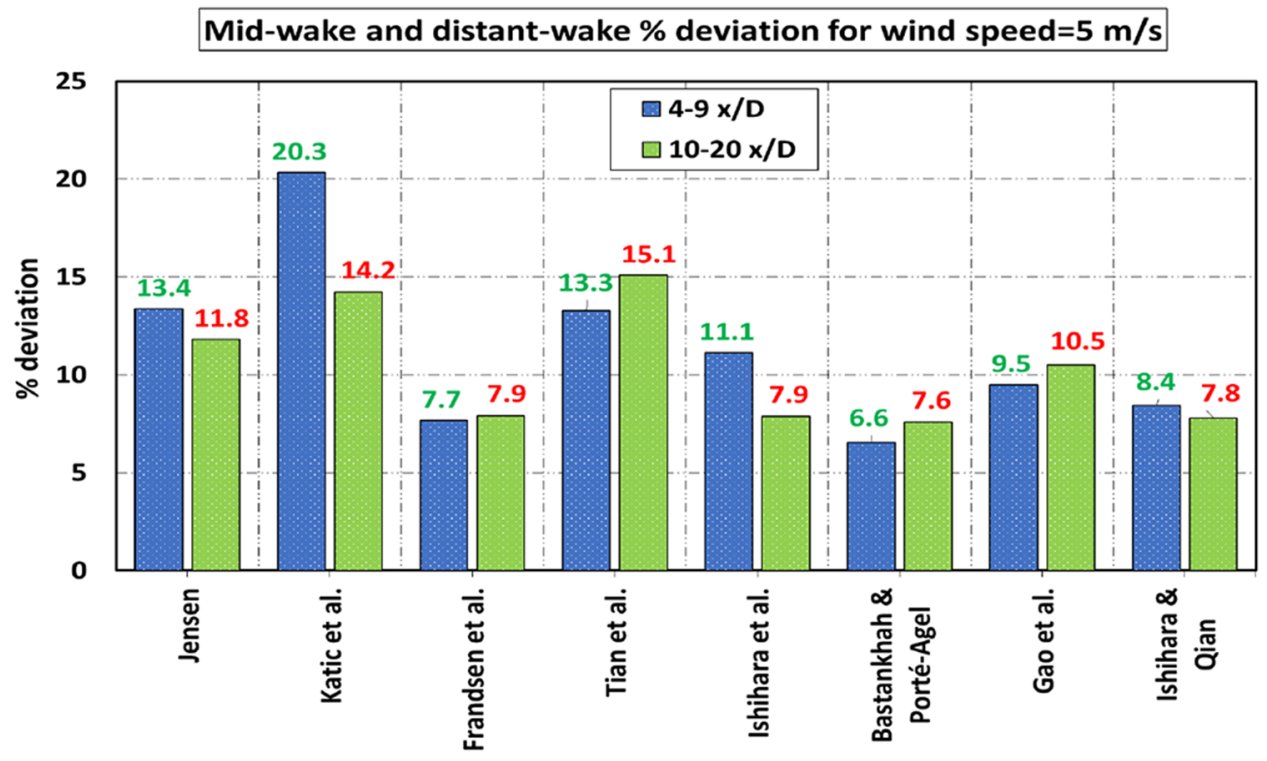

Fig. 5. Average percentage deviation of each wake model for upstream wind speed value equal to $5 \mathrm{~m} / \mathrm{s}$.

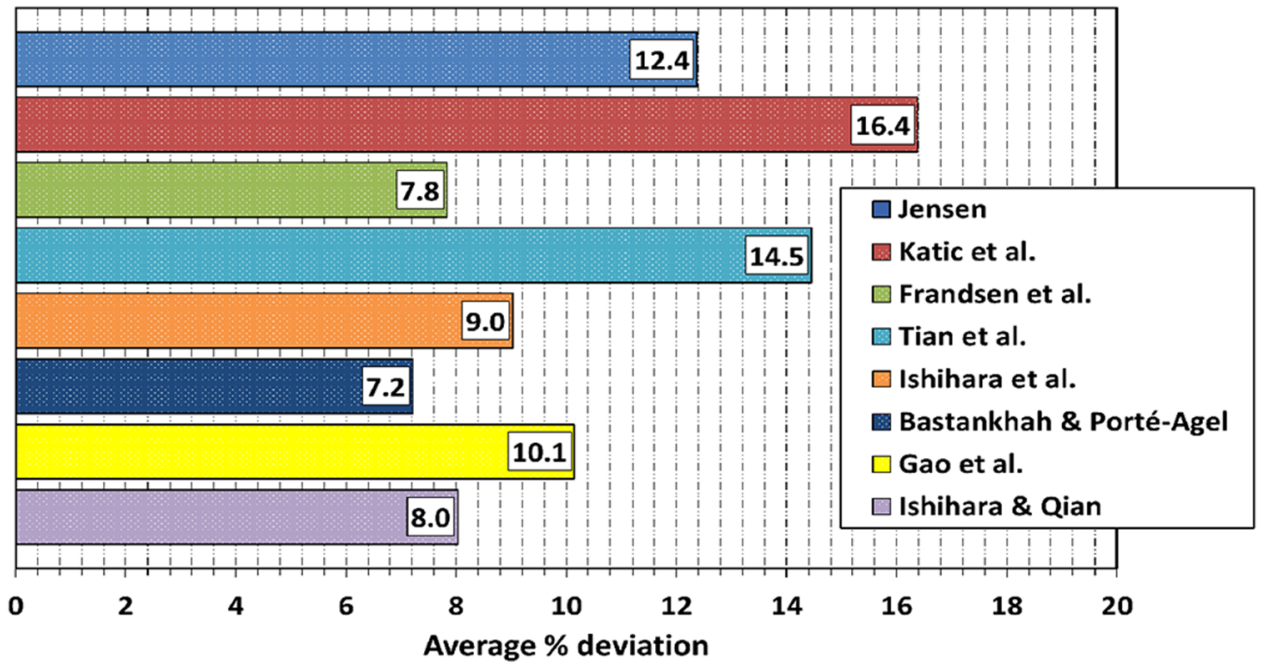

Fig. 6. Average percentage deviation of each wake model in the entire far-wake region for upstream wind speed value equal to $5 \mathrm{~m} / \mathrm{s}$.

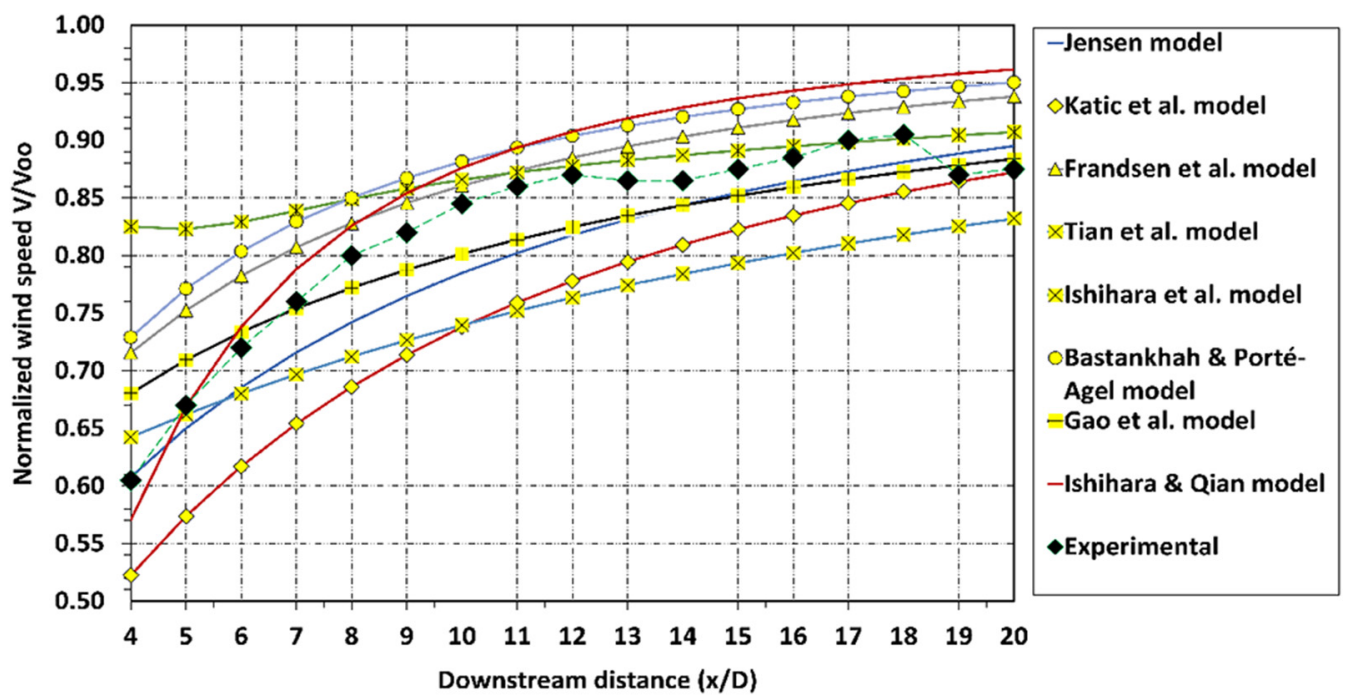

Fig. 7. Experimental and calculated wake development for upstream wind speed value equal to $13 \mathrm{~m} / \mathrm{s}$. 


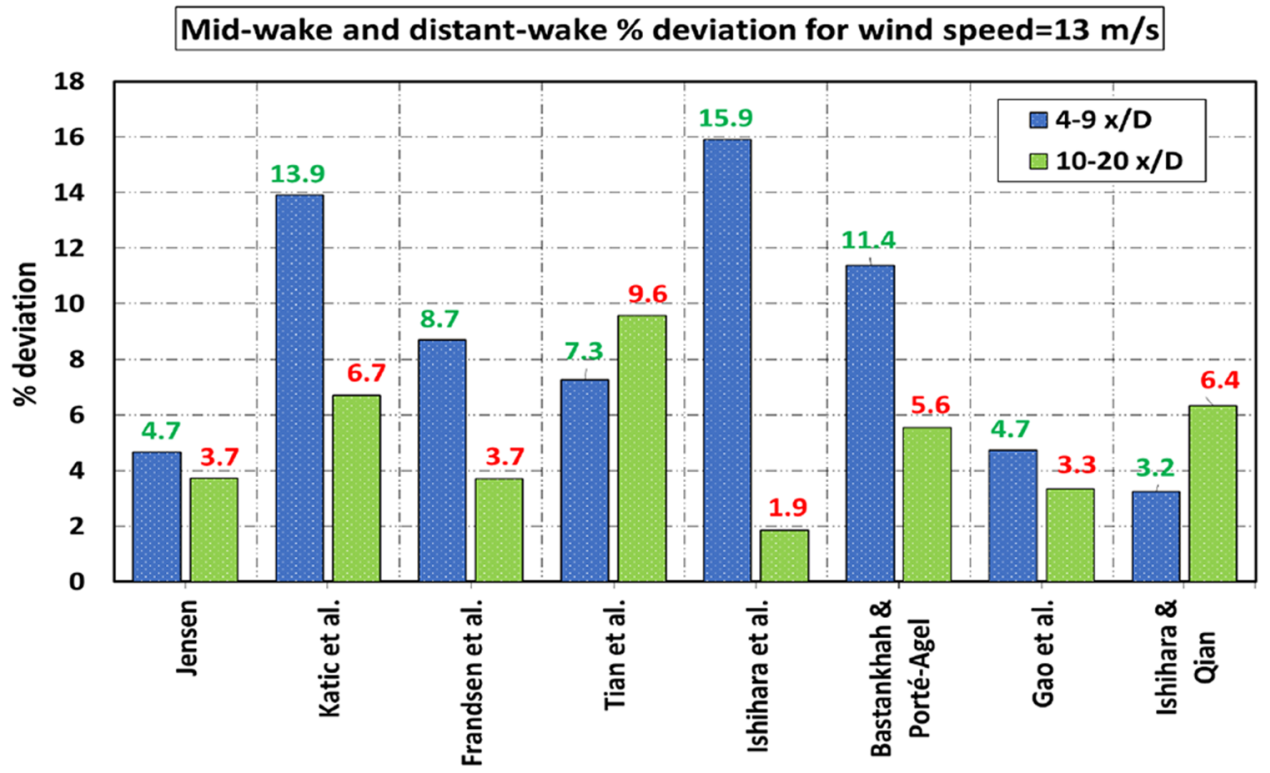

Fig. 8. Average percentage deviation of each wake model for upstream wind speed value equal to $13 \mathrm{~m} / \mathrm{s}$.

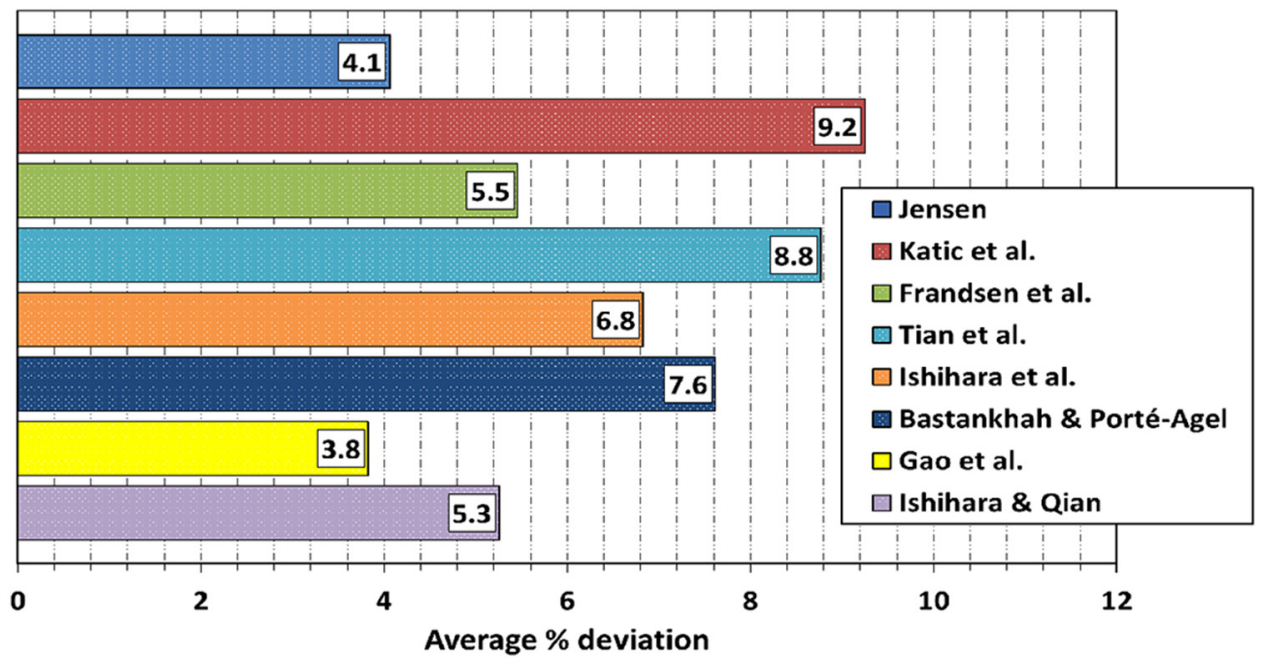

Fig. 9. Average percentage deviation of each wake model in the entire far-wake region for upstream wind speed value equal to $13 \mathrm{~m} / \mathrm{s}$.

the two far-wake sub-regions, exceeding 12\%. More precisely, the corresponding percentage deviations lie in the range $[2-7 \%]$, highlighting the sufficiently good experimental measurements reproduction.

Except the Jensen and the Gao et al. models, great differences are present between the average percentage deviations recorded in the mid-wake and the distant-wake sub-regions (Fig. 8), being in the order of magnitude greater than $2 \%$. The greatest discrepancy, equal to $14 \%$, is recorded for the Ishihara et al. model.

As a direct repercussion of the higher to the previous upstream wind speed value case investigated, an improved models' performance is noted. More precisely, most of the wake models imvestigated, except the ones of Katic et al. and Tian et al., demonstrate average percentage deviations less than $8 \%$ for the entire far-wake region (Fig. 9). The best performance is recorded for the Jensen and Gao et al. models that present an average percentage deviation roughly equal to $4 \%$. On the contrary, the average percentage deviation of the Katic et al. and the Tian et al. models approximates $9 \%$.

\subsection{Prediction performance evaluation for wind speed value equal to $17 \mathrm{~m} / \mathrm{s}$}

Similar to the previous wind speed value case, the wind speed value examined in this Section lies in the WT's power curve constant power segment. Most of the wake models investigated are characterized by acceptable percentage deviations from the experimental measurements (Fig. 10), being less than $9 \%$, for the entire far-wake region, except for downstream distance value in the range between four and seven "x/D". Furthermore, the wake models outcome deviation from the experimental measurements decreases 


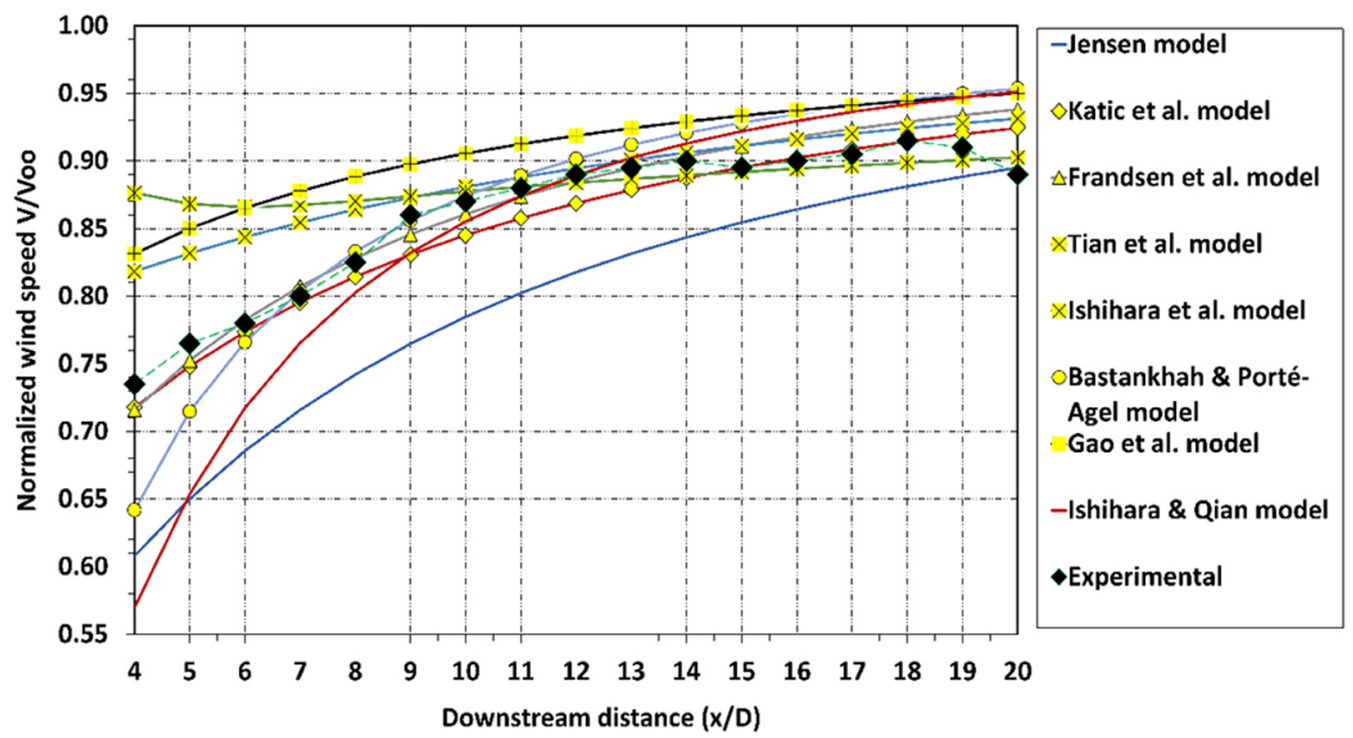

Fig. 10. Experimental and calculated wake development for upstream wind speed value equal to $17 \mathrm{~m} / \mathrm{s}$.

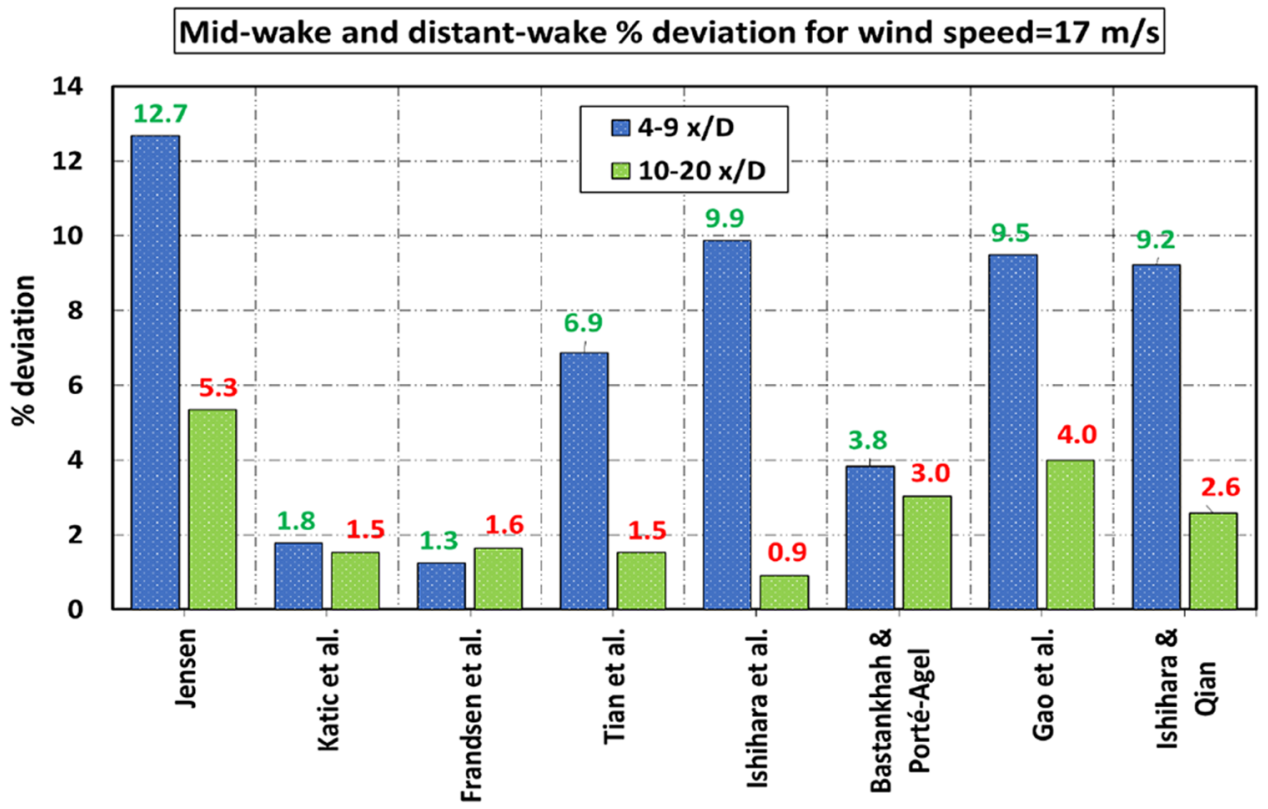

Fig. 11. Average percentage deviation of each wake model for upstream wind speed value equal to $17 \mathrm{~m} / \mathrm{s}$.

with the downstream distance increase in the mid-wake sub-region, whereas it is almost held constant in the order of $2-4 \%$ in the distant-wake sub-region, emphasizing the wake models' great performance. It is only the Jensen model that presents percentage deviation greater than $8 \%$ in the distinct limit between the mid-wake and the distantwake sub-regions.

The increased upstream wind speed value with regards to the previous wind speed value case retains the turbulence intensity value at high levels. Except the Katic et al., the Bastankhah \& Porté-Agel and the Frandsen et al. models, the discrepancies recorded between the average percentage deviations in the mid-wake and the distantwake sub-regions (Fig. 11) are found in the range [5-9\%].
On the other hand, for the three specific models, the relevant differences between the average percentage deviations in the two aforementioned sub-regions are equal to $0.3 \%, 0.8 \%$ and $0.3 \%$ correspondigly, pinpointing their exceptional performance.

The overall outstanding prediction performance for all wake models examined is further validated by their average percentage deviation values in the entire far-wake region (Fig. 12). In fact, the upper limit for all wake models' average prediction outcome is the value of almost $8 \%$ that characterizes the Jensen wake model. It is also characteristic that the Katic et al. and the Frandsen et al. models are characterized by average percentage deviations approximating $1.5 \%$. 


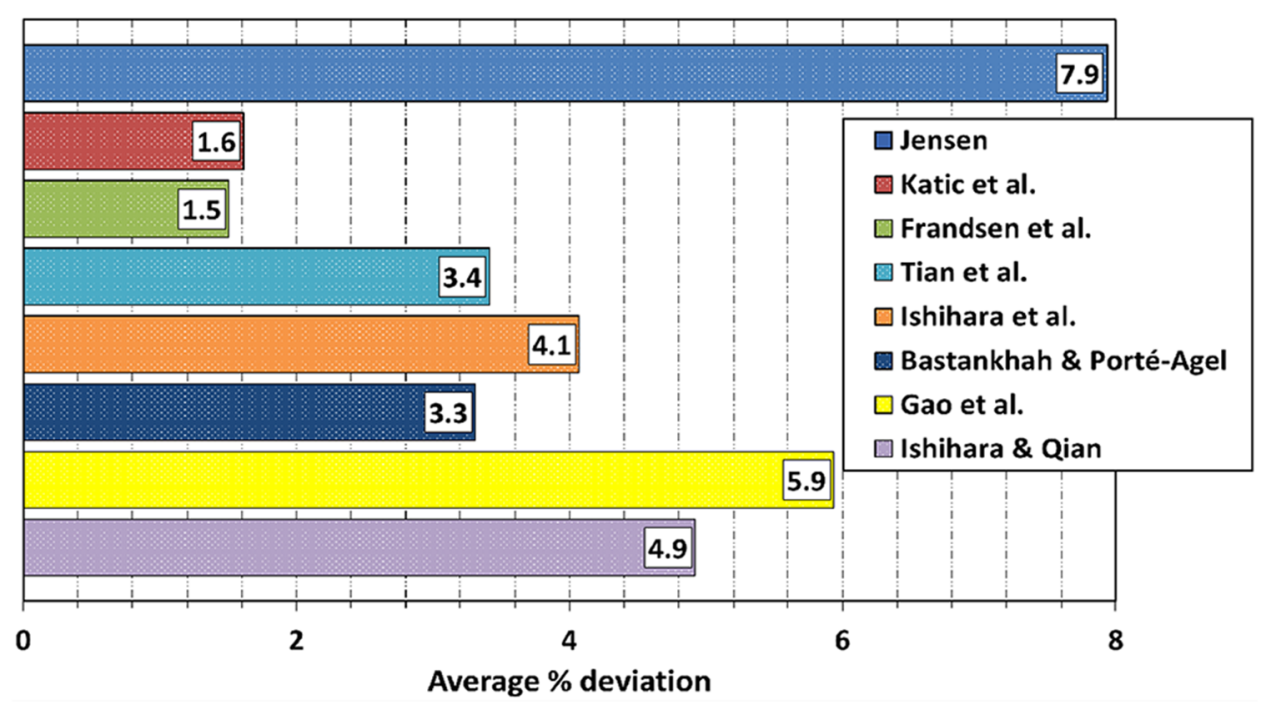

Fig. 12. Average percentage deviation of each wake model in the entire far-wake region for upstream wind speed value equal to $17 \mathrm{~m} / \mathrm{s}$.

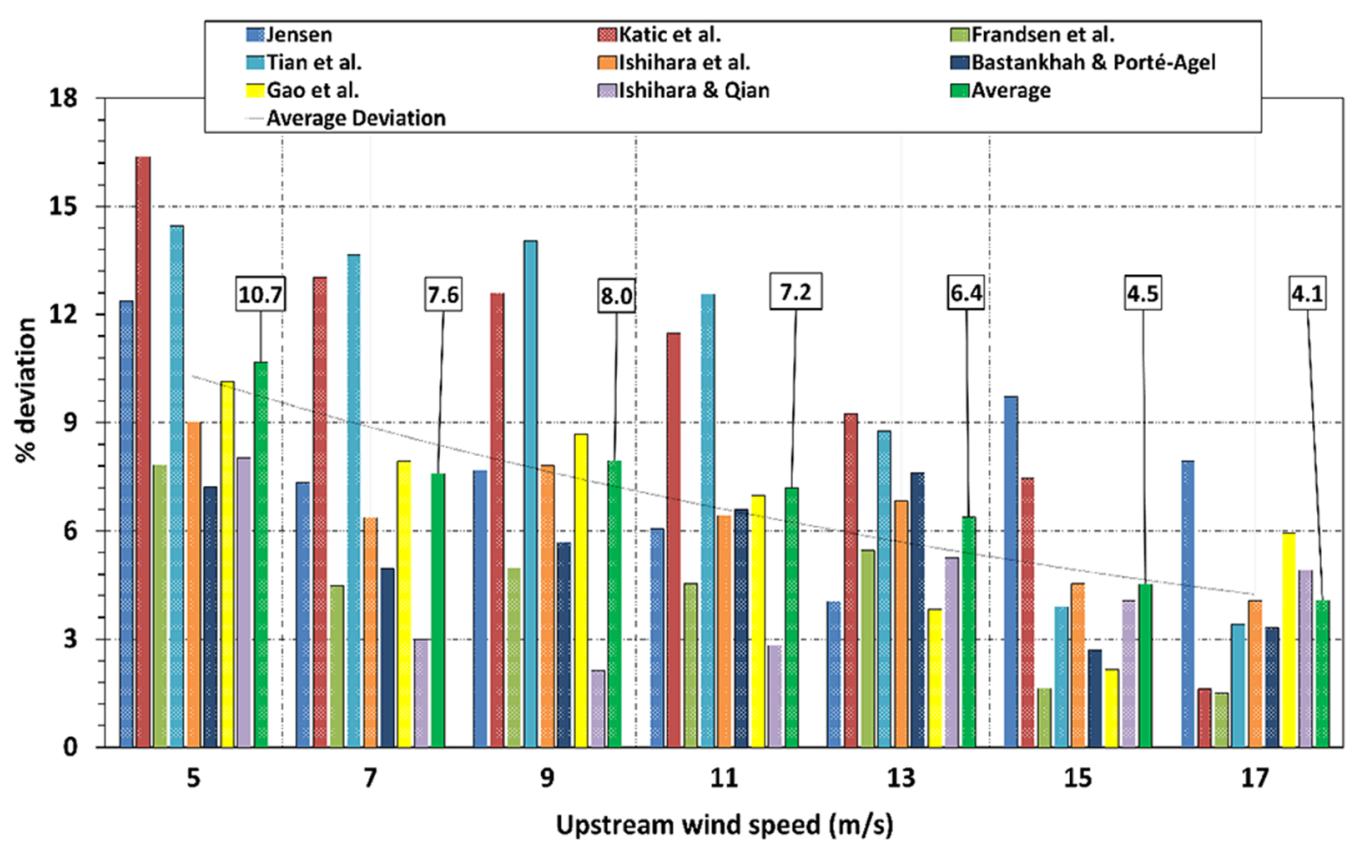

Fig. 13. Percentage deviation per wake model and upstream wind speed in the entire far-wake region. Note: The green column (with the data labels) depicts the average percentage deviation of all wake models investigated.

\section{Prediction performance evaluation for the entire wind speed value range}

Following the previous analysis, it can be concluded that great discrepancies are noted between the wake models prediction performance regarding the wake evolution rate. Depending on their input parameters and their inherent calculations, some wake models overestimate this rate, with others underestimating it. Figure 13 presents the percentage deviation variation per wake model and upstream wind speed, with the cumulative average percentage deviations being depicted in Figure 14.

The prediction performance deviation is inversely proportionate to the upstream wind speed for the majority of the numerical wake models examined (Fig. 13). The least discrepancy between the minimum and the maximum calculated values in the entire far-wake region is noted for the Bastankhah \& Porté-Agel and the Ishihara et al. models outcome, being in the order of almost 5\%. The 


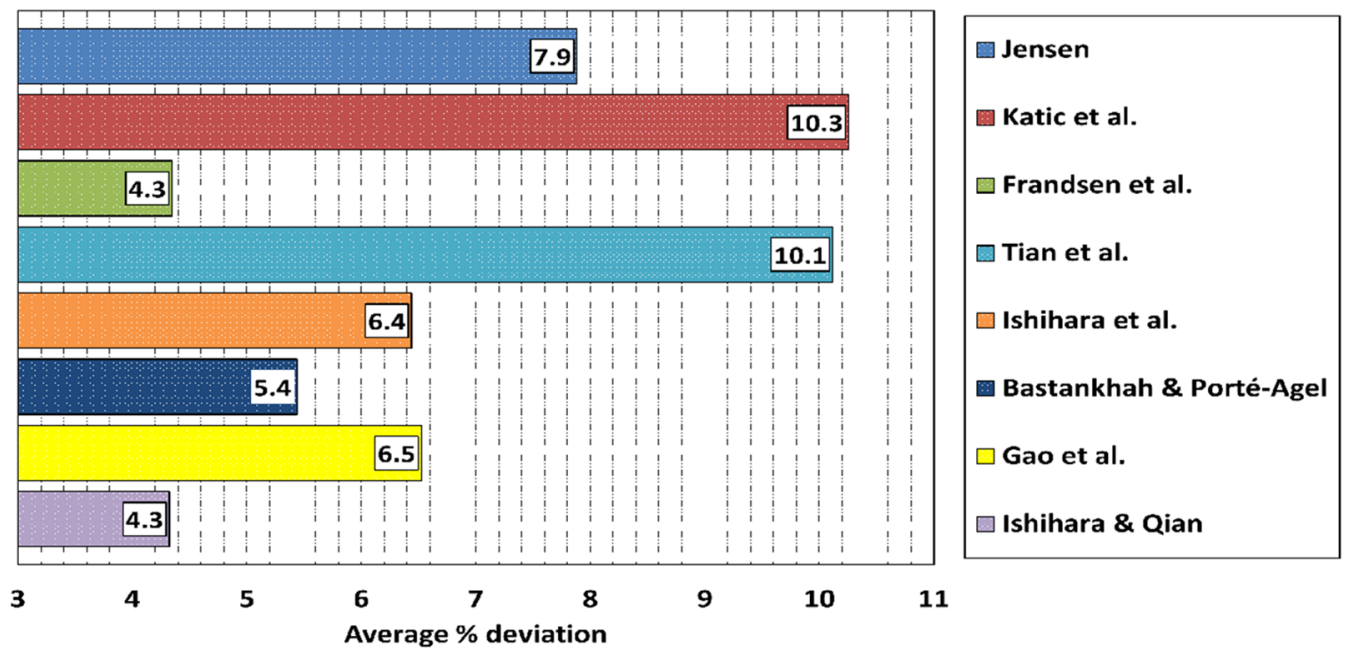

Fig. 14. Cumulative average percentage deviations for all wake models.

specific noteworthy remark can be mostly ascribed to the specific models' reliance on the turbulence intensity value. On the other hand, the greatest difference is recorded for the Katic et al. model.

In the same context, owning to the great percentage deviation values that characterize the Jensen and the Gao et al. models in the upstream wind speed range $[5-9] \mathrm{m} / \mathrm{s}$ as well as the Tian et al. and the Katic et al. models in the upstream wind speed range $[5-13] \mathrm{m} / \mathrm{s}$ (Fig. 13), an increased average percentage deviation value, almost equal to $7 \%$, is generated for the entire far-wake region. The aforementioned value is greater than the relevant one corresponding to five out of eight numerical wake models investigated (Fig. 14). To be more specific, the Frandsen et al., the Ishihara et al., the Bastankhah \& Porté-Agel, the Gao et al. and the Ishihara \& Qian models are characterized by improved results, with their average percentage deviations being $4.3 \%, 6.4 \%, 5.4 \%, 6.5 \%$ and $4.3 \%$ respectively. In its turn, the average percentage deviation of the classical Jensen model is marginally higher than the average value and is equal to $7.9 \%$.

\section{Conclusions}

The current work conducts a thorough comparative analysis of eight well-established numerical, semi-empirical and analytical wake models, developed to represent the wind speed downstream the Wind Turbines' (WTs) hub. The comparison has been carried out using benchmark experimental data retrieved from a pertinent scientific work.

The wake models accuracy is proportional to their ability in representing the experimental measurements. Among the numerical wake models examined, the ones deployed by Jensen, Frandsen et al., Bastankhah \& PortéAgel, Ishihara et al., Gao et al. and Ishihara \& Qian describe the experimental data with admissible accuracy, as their average percentage deviation does not exceed $8 \%$ maximum. The validity of the last remark also for the Jensen model justifies its wide utilization (with optimized equations) for industrial software deployment, exploiting in this way its easy implementation, restricted number of necessary input data and lack of complexity.

The "top-hat" shaped nature of the wake models examined, excluding the ones of Ishihara \& Qian and Bastankhah \& Porté-Agel, can lead to increased error rates in wind speed prediction and the subsequent pertinent energy estimation calculations. Under this scope, further investigation of the influence that dedicated parameters, such as the mixed turbulence intensity or the wind direction, can have on the wake models' constituents and outcome is considered crucial and will shed light on the repercussions that the wake effect has on the pertinent wind farm's micrositing optimization. In the same context, the limited calculated deviations of the Ishihara \& Qian and the Bastankhah \& Porté-Agel models render their further evolution quite critical and useful. Finally, a relevant validation of benchmark data, either experimental measurements or simulation results, with wake superposition models will set the foundations for a better comprehension of the interaction between successive WTs' rows and the wake development.

Recapitulating, using available experimental data, the present work has demonstrated that there exist numerical, semi-empirical or analytical wake models capable of predicting the wake evolution characteristics downstream of a contemporaneous WT with sufficient precision (maximum percentage deviation equal to $8 \%$ ). Special emphasis has been given on the wake models' performance evaluation in the two sub-regions of the far-wake area in order to elucidate their impact on the relevant wake evolution and stand out as a supporting tool for wind power project developers. The significantly low computational burden that characterizes the numerical, analytical and semi-empirical wake models examined along with their trustworthy accuracy justify their integration in WT controllers' strategies for a preliminary wind farm's 
micrositing assessment. For a more comprehensive analysis and investigation, the combined utilization of these models with more sophisticated simulation algorithms could be justified as rational.

\section{References}

1. A.S. Darwish, R. Al-Dabbagh, Wind energy state of the art: present and future technology advancements, Renew. Energy Environ. Sustain. 5, 7 (2020)

2. J.Y. Jin, R. Ghani, M.S. Virk, Wind turbine wake effects on wind resource assessments - a case study, E3S Web Conf. 186, $03003(2020)$

3. Y. Wang, Q. Hu, L. Li, A.M. Foley, D. Srinivasan, Approaches to wind power curve modeling: a review and discussion, Renew. Sustain. Energy Rev. 116 (2019)

4. M.J. Churchfield, A review of wind turbine wake models and future directions, in Proceedings of 2013 North American Wind Energy Academy (NA WEA) Symposium (2013) p. 1-20

5. N.P. Dufresne, M. Wosnik, Velocity deficit and swirl in the turbulent wake of a wind turbine, Mar. Technol. Soc. J. 47, 4 (2013)

6. A. Rasheed, K. Sørli, R. Holdahl, T. Kvamsdal, A multiscale approach to micrositing of wind turbines, Energy Proc. 14 (2012)

7. J.K. Kaldellis, M. Kapsali, E. Kaldelli, E. Katsanou, Comparing recent views of public attitude on wind energy, photovoltaic and small hydro applications, Renew. Energy 52 (2013)

8. L. Tian, W. Zhu, W. Shen, Y. Song, N. Zhao, Prediction of multi-wake problems using an improved Jensen wake model, Renew. Energy 102B (2017)

9. G.C. Larsen, H.A. Madsen, F. Bingöl, J. Mann, S. Ott, J.N. Sørensen, V. Okulov, N. Troldborg, M. Nielsen, K. Thomsen, T.J. Larsen, R. Mikkelsen, Dynamic wake meandering modeling, Ris $\varnothing$ National Laboratory, Denmark 1607 (2007)

10. A.A. Veisi, M.H.S. Mayam, Large Eddy Simulation of flow around a single and two in-line horizontal-axis wind turbines, Energy 121 (2017)

11. H. Hu, Z. Yang, P. Sarkar, Dynamic wind loads and wake characteristics of a wind turbine model in an atmospheric boundary layer wind, Exp. Fluids 52, 5 (2012)

12. J.K. Kaldellis, D. Zafirakis, The influence of technical availability on the energy performance of wind farms: overview of critical factors and development of a proxy prediction model, J. Wind Eng. Ind. Aerodyn. 115 (2013)

13. X. Gao, H. Yang, L. Lin, Optimization of wind turbine layout position in a wind farm using a newly-developed twodimensional wake model, Appl. Energy 174 (2016)

14. E. Barlas, W.J. Zhu, W.Z. Shen, M. Kelly, S. Andersen, Effects of wind turbine wake on atmospheric sound propagation, Appl. Acoust. 122 (2017)

15. A. Laratro, M. Arjomandi, R. Kelso, B. Cazzolato, A discussion of wind turbine interaction and stall contributions to wind farm noise, J. Wind Eng. Ind. Aerodyn. 127 (2014)

16. D. Heimann, Y. Käsler, G. Gross, The wake of a wind turbine and its influence on sound propagation, Meteorolog. Zeitsch. $20(2011)$

17. I.F.S.A. Kabir, E.Y.K. Ng, Effect of different atmospheric boundary layers on the wake characteristics of NREL phase VI wind turbine, Renew. Energy 130 (2019)
18. S. Rockel, J. Peinke, M. Hölling, R.B. Cal, Dynamic wake development of a floating wind turbine in free pitch motion subjected to turbulent inflow generated with an active grid, Renew. Energy 112, 2 (2017)

19. D.A. Katsaprakakis, D.G. Christakis, Wind parks design, including representative case studies, in Comprehensive Renewable Energy, edited by A. Sayigh, J.K. Kaldellis (Oxford, Elsevier, 2012)

20. M. Kapsali, J.K. Kaldellis, Offshore wind power basics, in Comprehensive Renewable Energy, edited by A. Sayigh, J.K. Kaldellis (Oxford, Elsevier, 2012)

21. F. Porté-Agel, M. Bastankhah, S. Shamsoddin, Windturbine and wind-farm flows: a review, Boundary-Layer Meteorol. 174 (2019)

22. B. Sanderse, Aerodynamics of wind turbine wakes Literature review (Energy Research Centre of the Netherlands, 2009)

23. F. Zahle, N.N. Sørensen, On the influence of far-wake resolution on wind turbine flow simulations, J. Phys.: Conf. Ser. 75, 1 (2007)

24. J.K. Kaldellis, P. Triantafyllou, P. Stinis, Critical evaluation of Wind Turbines' analytical wake models, Renew. Sustain. Energy Rev. 144C, 110991 (2021)

25. L. Tian, W. Zhu, W. Shen, N. Zhao, Z. Shen, Development and validation of a new two-dimensional wake model for wind turbine wakes, J. Wind Eng. Ind. Aerodyn. 137 (2015)

26. M. Talavera, F. Shu, Experimental study of turbulence intensity influence on wind turbine performance and wake recovery in a low-speed wind tunnel, Renew. Energy 107 (2017)

27. M.S. Adaramola, P.Å. Krogstad, Experimental investigation of wake effects on wind turbine performance, Renew. Energy 36, 8 (2011)

28. R.J. Barthelmie, S.C. Pryor, S.T. Frandsen, K.S. Hansen, J.G. Schepers, K. Rados, W. Schlez, A. Neubert, L.E. Jensen, S. Neckelmann, Quantifying the impact of wind turbine wakes on power output at offshore wind farms, J. Atmos. Ocean. Technol. 27, 8 (2010)

29. J.F. Ainslie, Calculating the flowfield in the wake of wind turbines, J. Wind Eng. Ind. Aerodyn. 27, 1-3 (1988)

30. D. Gallacher, G. More, Lidar measurements and visualisation of turbulence and wake decay length, in Proceedings of Europe's Premier Wind Energy Event (EWEA), 10-13 March 2014, p. $1-10$

31. T. Ishihara, G.W. Qian, A new Gaussian-based analytical wake model for wind turbines considering ambient turbulence intensities and thrust coefficient effects, J. Wind Eng. Ind. Aerodyn. 177 (2018)

32. H.S. Dhiman, D. Deb, A.M. Foley, Bilateral Gaussian wake model formulation for wind farms: a forecasting based approach, Renew. Sustain. Energy Rev. 127 (2020)

33. H. Sun, H. Yang, Numerical investigation of the average wind speed of a single wind turbine and development of a novel three-dimensional multiple wind turbine wake model, Renew. Energy 147, 1 (2020)

34. L. Parada, C. Herrera, P. Flores, V. Parada, Wind farm layout optimization using a Gaussian-based wake model, Renew. Energy 107 (2017)

35. R. Shakoor, M.Y. Hassa, A. Raheem, Y.K. Wu, Wake effect modeling: a review of wind farm layout optimization using Jensen's model, Renew. Sustain. Energy Rev. 58 (2016) 
36. H. Sun, X. Gao, H. Yang, A review of full-scale wind-field measurements of the wind-turbine wake effect and a measurement of the wake-interaction effect, Renew. Sustain. Energy Rev. 132, 110042 (2020)

37. E. Machefaux, G.C. Larsen, J.P. Murcia Leon, Engineering models for merging wakes in wind farm optimization applications, J. Phys: Conf. Ser. 625 (2015)
38. M. Gaumond, P.E. Réthoré, A. Bechmann, S. Ott, G.C Larsen, A. Pena, K.S. Hansen, Benchmarking of Wind Turbine Wake Models in Large Offshore Wind Farms (DTU Wind Energy, 2012)

39. S. Jeon, B. Kim, J. Huh, Comparison and verification of wake models in an onshore wind farm considering single wake condition of the $2 \mathrm{MW}$ wind turbine, Energy 93, 2 (2015)

Cite this article as: Panagiotis Triantafyllou, John K. Kaldellis, Wind turbine wake models' evaluation for different downstream locations, Renew. Energy Environ. Sustain. 6, 40 (2021) 